\title{
Riemann normal coordinates, Fermi reference system and the geodesic deviation equation
}

\begin{abstract}
Alexander I. Nesterov *
We obtain the integral formulae for computing the tetrads and metric components in Riemann normal coordinates and Fermi coordinate system of an observer in arbitrary motion. Our approach admits essential enlarging the range of validity of these coordinates. The results obtained are applied to the geodesic deviation in the field of a weak plane gravitational wave and the computation of plane-wave metric in Fermi normal coordinates.
\end{abstract}

KEY WORDS: Riemann coordinates, Fermi reference system

*Departamento de Física, Universidad de Guadalajara, Guadalajara, Jalisco, México; e-mail: nesterov@udgserv.cencar.udg.mx 


\section{INTRODUCTION}

The well known Riemann normal coordinates satisfy the conditions $\left.g_{\mu \nu}\right|_{p}=\eta_{\mu \nu}$ and $\left.\Gamma_{\nu \lambda}^{\mu}\right|_{p}=0$ at a point $p$, the origin of the coordinate system. In these cordinates the metric is presented in powers of the canonical parameter of the geodesics out from $p$. In 1922 Fermi [1] showed that it is possible to generalize the Riemann coordinates in such a way that the Christoffell symbols vanish along given any curve in a Riemannian manifold, leaving the metric there rectangular. For a geodesic curve Misner and Manasse [2] introduced a Fermi normal coordinates, which satisfy Fermi conditions along a given geodesic and calculated the first-order expansion of the connection coefficients and the second-order expansion of the metric in powers of proper distance normal to the geodesic. Their construction is a special case of Fermi coordinates defined by Synge [3]. The last ones form a natural coordinate system for a nonrotating accelerated observer.

In 1973 Misner, Thorn and Wheeler (MTW) [1] defined a Fermi coordinates for an accelerated and rotating observer and calculated the first-order expansion of the metric. Extending MTW work Mitskievich and Nesterov [5] and later Ni and Zimmerman [6] obtained the second-order expansion of the metric and the first-order expansions of the connection coefficients. Li and Ni [7.8] derived the third-order expansion of the metric and the second-order expansions of the connection coefficients.

The size of neighbourhood $V$, where Riemann and Fermi normal coordinates do not involve singularaties, is determined by conditions

$$
u \ll \min \left\{\frac{1}{\mid \stackrel{0}{R_{\alpha \gamma \delta \beta} \mid 1 / 2}}, \frac{\left|\stackrel{0}{R}_{\alpha \gamma \delta \beta}\right|}{\left|\stackrel{0}{R}_{\alpha \gamma \delta \beta, \mu}\right|}\right\}
$$

where $u$ is a canonical parameter along the geodesics from the origin of coordinates. The first condition $u \ll \mid \stackrel{0}{R}_{\alpha \gamma \delta \beta}$ $\left.\right|^{-1 / 2}$ determines the size of $V$ where the curvature has not yet caused geodesics to cross each other. The second condition determines the domain where the curvature does not change essentially. For instance, for gravitational waves with wavelength $\lambda$ the Riemann tensor is $\sim A \exp (i k x) / \lambda^{2}$, where $A$ is the dimensionless amplitude. This yields

$$
u \ll \min \{\lambda / \sqrt{A}, \quad \lambda\} .
$$

Generally it is assumed $A \leq 10^{-18}$. This means that the size of $V$ is restricted by $u_{0} \ll \lambda$. So the application of Riemann (or Fermi) coordinates to the modern experiments may be very restrictive since $\lambda$ is often supposed being in the order of $300 \mathrm{~km}$. Thus for enlarging the range of validity by a factor $1 / \sqrt{A}$ (which is about $10^{9}$ in our example) it is necesary to take into account derivatives of any order of the Riemann tensor. Some years ago Marzlin [9] has considered Fermi coordinates for weak gravitational field $g_{\mu \nu}=\eta_{\mu \nu}+h_{\mu \nu},\left|h_{\mu \nu}\right| \ll 1$ and dervived the metric as a Taylor expansion valid to all orders in the geodesic distance from the world line of Fermi observer.

Here we develop a new approach to Riemann and Fermi coordinates, which admits enlarging the range of validity of these coordinates up to $u \ll\left|\stackrel{0}{R}_{\alpha \gamma \delta \beta}\right|^{-1 / 2}$. The point is to use the integral formulae. Recently this approach has been used to calculate a geometric phase shift for a light beam propagating in the field of a weak gravitational wave [10,11.

The paper is organized as follows. In Section 2 we obtain the integral formulae for computing the tetrad and metric components in Riemann normal and Fermi coordinates. In Section 3 we compute the plane-wave metric in Fermi normal coordinates and analyse the geodesic deviation equation for this case.

In this paper we use the space-time signature $(+,-,-,-)$; Greek indices run from 0 to 3 , Latin $a, b, c$ from 1 to 2 and $i, j, k$ from 1 to 3 .

\section{GENERAL RESULTS}

\section{A. Riemann normal coordinates}

For arbitrary point $p_{0}$ in some neighbourhood $V\left(p_{0}\right)$ there is the unique geodesic $\gamma(u)$ connecting $p_{0}$ and $p$ which, using exponential mapping, we may write as $p=\exp _{\gamma(u)}(u \xi)$, where $\xi=\partial / \partial u=\xi^{\alpha} e_{(\alpha)}$, $u$ being the canonical parameter while basis is parallely propagated along $\gamma(u)$. The Riemann normal coordinates with the origin at the point $p_{0}$, are defined as $X^{\alpha}=u \xi^{\alpha}$, where $u \xi=\exp _{\gamma(u)}^{-1}(p)$. The following basic equations summarize the most important properties of the Riemannian normal coordinates: 


$$
\begin{aligned}
& \mathbf{e}_{(\alpha)}\left(p_{0}\right)=\left(\partial / \partial X^{\alpha}\right)_{\mathrm{p}_{0}}=\left(\Lambda_{\alpha}^{\beta} \partial / \partial x^{\beta}\right)_{p_{0}}, \Lambda_{\alpha}{ }^{\beta}=\left(\partial x^{\beta} / \partial X^{\alpha}\right)_{\mathrm{p}_{0}} \\
& \stackrel{0}{\Gamma}_{\beta \gamma}^{\alpha}=0, \Gamma_{\beta \gamma}^{\alpha} X^{\beta} X^{\gamma}=0, \quad \Gamma_{\beta \gamma}^{\alpha}=-\frac{2}{3} \stackrel{0}{R^{\alpha}}(\beta \gamma) \delta X^{\delta}+\cdots,
\end{aligned}
$$

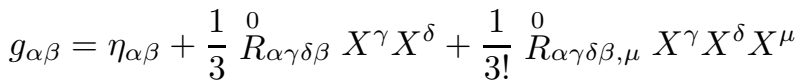

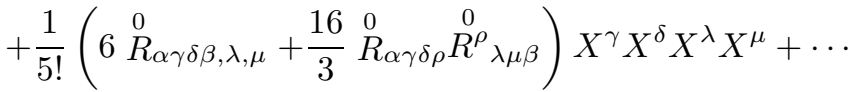

The size of neighbourhood $V$, where these coordinates do not involve singularaties, is determined by conditions

$$
u \ll \min \left\{\left.\frac{1}{\mid \stackrel{0}{R} \alpha \gamma \delta \beta}\right|^{1 / 2}, \frac{\mid \stackrel{0}{R} \frac{0}{R_{\alpha \gamma \delta \beta} \mid}}{|\stackrel{R}{\alpha \gamma \delta \beta, \mu}|}\right\} .
$$

The first condition $u \ll\left|\stackrel{0}{R}_{\alpha \gamma \delta \beta}\right|^{-1 / 2}$ determines the size of $V$ where the curvature has not yet caused geodesics to cross each other. The second condition determines the domain where the curvature does not change essentially.

For studing Riemann and Fermi normal coordinates one useful technique is the geodesic deviation equation [2]. Further we apply it for our computation. Let us consider a point $p_{0}$ and a frame $\left\{\mathbf{e}_{(\alpha)}\left(p_{0}\right)\right\}$ in this point. For arbitrary geodesic $\gamma(u)$ out from $p_{0}$ the equation of geodesic deviation has the following form

$$
\nabla_{\xi}^{2} \eta+\mathrm{R}(\xi, \eta) \xi=0
$$

where $\xi=\partial / \partial u$ is tangent vector to $\gamma(u), \eta$ is vector of deviation and R is operator of curvature. We suppose that the basis $\mathbf{e}_{(\alpha)}$ is parallely propagated along $\gamma(u)$. In tetrad components the equation (5) takes the form

$$
\frac{d^{2} \eta^{(\alpha)}}{d u^{2}}=R_{(\beta)(\gamma)(\delta)}^{(\alpha)} \xi^{(\beta)} \xi^{(\gamma)} \eta^{(\delta)}
$$

where $\eta^{(\alpha)}=\eta^{\beta} e_{\beta}^{(\alpha)}$, etc.

In Riemann normal coordinates there are natural deviation vectors $\eta_{\alpha}=u \delta_{\alpha}^{\beta} \partial / \partial X^{\beta}$ satisfying Eq.(5) [2]. Then (5) yields

$$
\frac{d^{2} e_{\beta}^{(\alpha)}}{d u^{2}}+\frac{2}{u} \frac{d e_{\beta}^{(\alpha)}}{d u}=e_{\lambda}^{(\alpha)} R_{\gamma \delta \beta}^{\lambda} \xi^{\gamma} \xi^{\delta} .
$$

We solve this equation by succesive approximations [12]. Let us write (7) in the equivalent integral form

$$
e_{\beta}^{(\alpha)}=\delta_{\beta}^{\alpha}+\frac{1}{u} \int_{0}^{u} d \tau \int_{0}^{\tau} d \tau^{\prime} \tau^{\prime} e_{\lambda}^{(\alpha)} R_{\gamma \delta \beta}^{\lambda} \xi^{\gamma} \xi^{\delta},
$$

where we assume $e_{\beta}^{(\alpha)}=\delta_{\beta}^{\alpha}$ at the origin of Riemann coordinates. Now we start with

$$
e_{\beta}^{(\alpha)} \approx e_{0 \beta}^{(\alpha)}=\delta_{\beta}^{\alpha}
$$

To improve this first approximation we feed back $e_{0 \beta}^{(\alpha)}$ into the integral (8) getting

$$
e_{1 \beta}^{(\alpha)}=\delta_{\beta}^{\alpha}+\frac{1}{u} \int_{0}^{u} d \tau \int_{0}^{\tau} d \tau^{\prime} \tau^{\prime} R_{\gamma \delta \beta}^{\alpha} \xi^{\gamma} \xi^{\delta},
$$

Repeteang this process by substituting the new $e_{i \beta}^{(\alpha)}$ back into Eq. (8), we find our solution $e_{\beta}^{(\alpha)}$ as infinite series

$$
\begin{aligned}
& e_{\beta}^{(\alpha)}=\sum_{n=0}^{\infty} \stackrel{(n)(\alpha)}{e_{\beta}} \\
& \stackrel{(0)(\alpha)}{e_{\beta}}=\delta_{\beta}^{\alpha} \\
& \stackrel{(1)(\alpha)}{e_{\beta}}=\frac{1}{u} \int_{0}^{u} d \tau \int_{0}^{\tau} d \tau^{\prime} \tau^{\prime} \stackrel{(0)(\alpha)}{e}_{\lambda}^{(\alpha)} R_{\gamma \delta \beta}^{\lambda} \xi^{\gamma} \xi^{\delta}, \\
& \stackrel{(2)(\alpha)}{e_{\beta}}=\frac{1}{u} \int_{0}^{u} d \tau \int_{0}^{\tau} d \tau^{\prime} \tau^{\prime} \stackrel{(1)(\alpha)}{e}_{\lambda} R_{\gamma \delta \beta}^{\lambda} \xi^{\gamma} \xi^{\delta}, \\
& \stackrel{(n+1)(\alpha)}{e_{\beta}}=\frac{1}{u} \int_{0}^{u} d \tau \int_{0}^{\tau} d \tau^{\prime} \tau^{\prime}{\stackrel{(n)(\alpha)}{e_{\lambda}}}^{\lambda} R_{\gamma \delta \beta}^{\lambda} \xi^{\gamma} \xi^{\delta} .
\end{aligned}
$$


Noting that the order of magnitude of functions $\stackrel{(n)(\alpha)}{e}_{\beta}$ is given in the neighbourhood $V$ by

$$
e_{\beta}^{(n)(\alpha)} \leq \frac{\left|R_{\alpha \gamma \delta \beta} X^{\gamma} X^{\delta}\right|_{\max }^{n}}{(2 n+1) ! !},
$$

we conclude that the radius of convergence of series (10) is determined by

$$
u_{0} \simeq\left\{\frac{1}{\left|R_{\alpha \gamma \delta \beta}\right|_{\max }^{1 / 2}}\right\} .
$$

Now using (710) one obtains for the components of orthonormal tetrad in Riemann coordinates the following integral formula:

$$
e_{\beta}^{(\alpha)}=\delta_{\beta}^{\alpha}+\frac{1}{u} \int_{0}^{u} d \tau \int_{0}^{\tau} d \tau^{\prime} \tau^{\prime} R_{\gamma \delta \beta}^{\alpha} \xi^{\gamma} \xi^{\delta}+\mathcal{O}\left(R^{2}\right)
$$

Remark 2.1. Integrating by parts one can write (11) as

$$
e_{\beta}^{(\alpha)}=\delta_{\beta}^{\alpha}+\int_{0}^{u} d \tau \int_{0}^{\tau} d \tau^{\prime} R^{\alpha}{ }_{\gamma \delta \beta} \xi^{\gamma} \xi^{\delta}-\frac{2}{u} \int_{0}^{u} d \tau \int_{0}^{\tau} d \tau^{\prime} \int_{0}^{\tau^{\prime}} d \tau^{\prime \prime} R^{\alpha}{ }_{\gamma \delta \beta} \xi^{\gamma} \xi^{\delta}+\mathcal{O}\left(R^{2}\right) .
$$

This form is more convenient for the comparison with the results obtained in Fermi coordinates (see Subsection B). For the covariant derivative of the tetrad we obtain the following useful formula (see Appendix A)

$$
\nabla_{\partial_{\lambda}} e_{(\nu)}^{\mu}=-\frac{1}{u} \int_{0}^{u} R^{\mu}{ }_{\nu \lambda \rho} \xi^{\rho} \tau d \tau+\mathcal{O}\left(R^{2}\right)
$$

With the aid of (11), it is straightforward to derive the metric in Riemann coordinates by using $g_{\alpha \beta}=\eta_{\mu \nu} e_{\alpha}^{(\mu)} e_{\beta}^{(\nu)}$. The result is

$$
g_{\alpha \beta}=\eta_{\alpha \beta}+\frac{2}{u} \int_{0}^{u} d \tau \int_{0}^{\tau} d \tau^{\prime} \tau^{\prime} R_{\alpha \gamma \delta \beta} \xi^{\gamma} \xi^{\delta}+\mathcal{O}\left(R^{2}\right)
$$

and can be rewritten by using Taylor expansion as

$$
g_{\alpha \beta}=\eta_{\alpha \beta}+\frac{1}{3} \stackrel{0}{R}_{\alpha \gamma \delta \beta} X^{\gamma} X^{\delta}+\frac{1}{3 !} \stackrel{0}{R}_{\alpha \gamma \delta \beta, \mu} X^{\gamma} X^{\delta} X^{\mu}+\frac{6}{5 !} \stackrel{0}{R}_{\alpha \gamma \delta \beta, \lambda, \mu} X^{\gamma} X^{\delta} X^{\lambda} X^{\mu}+\cdots,
$$

that agrees with Eq.(3).

In fact, we are interested in expressions which should involve (in the right-hand sides) the arbitrary chosen initial non-Riemann coordinates only. Then

$$
e_{\beta}^{(\alpha)}=\delta_{\beta}^{\alpha}+\frac{1}{u} \int_{0}^{u} d \tau \int_{0}^{\tau} d \tau^{\prime} \tau^{\prime} R_{\gamma \delta \sigma}^{\lambda} \frac{\partial X^{\alpha}}{\partial x^{\lambda}} \frac{\partial x^{\sigma}}{\partial X^{\beta}} \frac{\partial x^{\gamma}}{\partial X^{\kappa}} \frac{\partial x^{\delta}}{\partial X^{\rho}} \xi^{\kappa} \xi^{\rho}+\mathcal{O}\left(R^{2}\right) .
$$

The transformation from arbitrary coordinate system to Riemann one takes the form $x^{\mu}=x^{\mu}\left(p_{0}\right)+\Lambda_{\nu}^{\mu} X^{\nu}+\mathcal{O}(\Gamma)$ (or $\left.x^{\mu}=x^{\mu}\left(p_{0}\right)+\Lambda_{\nu}^{\mu} \xi^{\nu} u+\mathcal{O}(\Gamma)\right)$ and the equations (14), (15) can be written as

$$
\begin{gathered}
e_{\beta}^{(\alpha)}=\delta_{\beta}^{\alpha}+\frac{1}{u} \int_{0}^{u} d \tau \int_{0}^{\tau} d \tau^{\prime} \tau^{\prime} R^{\lambda}{ }_{\gamma \delta \sigma} \Lambda_{\lambda}^{-1 \alpha} \Lambda_{\kappa}{ }^{\gamma} \Lambda_{\rho}{ }^{\delta} \Lambda_{\beta}{ }^{\sigma} \xi^{\kappa} \xi^{\rho}+\mathcal{O}(R), \\
g_{\alpha \beta}=\eta_{\alpha_{\beta}}+\frac{2}{u} \int_{0}^{u} d \tau \int_{0}^{\tau} d \tau^{\prime} \tau^{\prime} R_{\lambda \gamma \delta \sigma} \Lambda_{\alpha}^{\lambda} \Lambda_{\kappa}{ }^{\gamma} \Lambda_{\rho}{ }^{\delta} \Lambda_{\beta}{ }^{\sigma} \xi^{\kappa} \xi^{\rho}+\mathcal{O}(R),
\end{gathered}
$$

where $R_{\gamma \delta \beta}^{\lambda}=R_{\gamma \delta \sigma}^{\lambda}\left(x^{\mu}\left(p_{0}\right)+\Lambda_{\nu}^{\mu} \xi^{\nu} \tau^{\prime}\right)$ while the integration is performed. 


\section{B. Fermi coordinates}

Let us consider the proper system of reference $\mathbf{e}_{(\alpha)}$ of a single observer which moves along a worldline $\gamma$ with an acceleration and rotation $\llbracket$

$$
\frac{D \mathbf{e}_{(\alpha)}}{d s}=\boldsymbol{\Omega} \cdot \mathbf{e}_{(\alpha)}
$$

where

$$
\Omega^{\mu \nu}=G^{\mu} \tau^{\nu}-G^{\nu} \tau^{\mu}+E^{\mu \nu \gamma \delta} \tau_{\gamma} \omega_{\delta}
$$

where $E^{\mu \nu \gamma \delta}$ is the axial tensor of Levi-Civita and $\tau^{\mu}$ is the unit tangent vector to the line $\gamma$; $G^{\mu}$ being the fouracceleration of observer, $\omega^{\delta}$ the four-rotation of the tetrad. Fermi coordinates are defined as $X^{\mu}=\delta_{0}^{\mu} s+\delta_{i}^{\mu} \xi^{i} u$ (see e.g. [2, (1). Here $s$ is proper time along the observer's world line $\gamma, \xi$ is a unit vector on $\gamma$ tangent to the spacelike geodesic starting from $\gamma$, ortogonal to it and parametrized by proper length $u$. In fact, $\xi$ describes the direction in which such a space-like geodesic goes, and it possesses only spatial components different from zero (the temporal coordinate is directed along $\gamma$ ). In Fermi coordinates, the covariant components of the corresponding orthonormal tetrad $e_{\mu}^{(\nu)}$ parallel transported along the spacelike geodesic, are represented as expansions (see Appendix B)

$$
\begin{array}{r}
e_{0}^{(\mu)}=\delta_{0}^{\mu}+\Omega^{\mu}{ }_{i} X^{i}+\frac{1}{2} \stackrel{0}{R}^{\mu}{ }_{i j 0} X^{i} X^{j}+\frac{1}{6} \stackrel{0}{R}_{0 i j 0} \Omega_{\Omega_{k}}^{\mu} X^{i} X^{j} X^{k}+\frac{1}{6} \stackrel{0}{R}^{\mu}{ }_{i j 0, k} X^{i} X^{j} X^{k}+\cdots, \\
e_{p}^{(\mu)}=\delta_{p}^{\mu}+\frac{1}{6}{\stackrel{0}{R^{\mu}}}^{\mu}{ }_{i j p} X^{i} X^{j}+\frac{1}{12} \stackrel{0}{R}^{0}{ }_{i j p} \Omega^{\mu}{ }_{k} X^{i} X^{j} X^{k}+\frac{1}{12} \stackrel{0}{R}^{\mu}{ }_{i j p, k} X^{i} X^{j} X^{k}+\cdots,
\end{array}
$$

where quantities of the type of $\stackrel{0}{Q}$ are taken on $\gamma$. The expansion up to $3 \mathrm{~d}$ order of the metric and up to 1st order of the connection coefficients (Christoffel symbols) is given by [5-8]

$$
\begin{aligned}
& g_{00}=1+2 \Omega_{0 p} X^{p}+\Omega_{\alpha k} \Omega^{\alpha}{ }_{p} X^{k} X^{p}+\stackrel{0}{R}{ }_{0 k p 0} X^{k} X^{p}+\stackrel{0}{R}_{\alpha i j 0} \Omega^{\alpha}{ }_{k} X^{i} X^{j} X^{k} \\
& +\frac{1}{3} \stackrel{0}{R}_{0 i j 0} \Omega_{0 k} X^{i} X^{j} X^{k}+\frac{1}{3} \stackrel{0}{R}_{0 i j 0, k} X^{i} X^{j} X^{k}+\cdots, \\
& g_{0 i}=\Omega_{i p} X^{p}+\frac{2}{3} \stackrel{0}{R} \text { iklo } X^{k} X^{l}+\frac{1}{4} \stackrel{0}{R}{ }_{i k l 0} \Omega_{0 m} X^{k} X^{l} X^{m}+\frac{1}{6} \stackrel{0}{R}{ }_{0 k l 0} \Omega_{i m} X^{k} X^{l} X^{m} \\
& +\frac{1}{6} \stackrel{0}{R} \text { iklm } \Omega_{n}^{m}{ }_{n} X^{k} X^{l} X^{n}+\frac{1}{4} \stackrel{0}{R} i k l 0, m X^{k} X^{l} X^{m}+\cdots, \\
& g_{i j}=\eta_{i j}+\frac{1}{3} \stackrel{0}{R} i k p j X^{k} X^{p}+\frac{1}{12}\left(\stackrel{0}{R}{ }_{i k l 0} \Omega_{j m} X^{k} X^{l} X^{m}+\stackrel{0}{R_{j k l 0}} \Omega_{i m} X^{k} X^{l} X^{m}\right) \\
& +\frac{1}{6} \stackrel{0}{R}{ }_{i k l j, m} X^{k} X^{l} X^{m}+\cdots, \\
& \Gamma_{0 \nu}^{\mu}=\Omega^{\mu}{ }_{\nu}+\left(\delta_{\nu}^{0} \dot{\Omega}^{\mu}{ }_{i}+\delta_{\nu}^{0} \Omega^{\mu}{ }_{\lambda} \Omega^{\lambda}{ }_{i}-\Omega^{0}{ }_{\nu} \Omega^{\mu}{ }_{i}+{\stackrel{0}{R^{\mu}}}_{\nu i 0}\right) X^{i}+\cdots, \\
& \Gamma_{i j}^{\mu}=-\frac{2}{3} \stackrel{0}{R}_{(i j) k}^{\mu} X^{k}+\cdots .
\end{aligned}
$$

For applications it is convenient to write the expansion for the metric tensor in the following form

$$
\begin{aligned}
& g_{00}=(1+\boldsymbol{G} \boldsymbol{X})^{2}-(\boldsymbol{\omega} \times \boldsymbol{X})^{2}+\stackrel{0}{R}_{0 k p 0} X^{k} X^{p}+\stackrel{0}{R}_{i j 0}^{l} \epsilon_{l k p} \omega^{p} X^{i} X^{j} X^{k} \\
& +\frac{4}{3} \stackrel{0}{R}_{0 i j 0} G_{k} X^{i} X^{j} X^{k}+\frac{1}{3} \stackrel{0}{R}_{0 i j 0, k} X^{i} X^{j} X^{k}+\cdots, \\
& g_{0 i}=-(\boldsymbol{\omega} \times \boldsymbol{X})_{i}+\frac{2}{3} \stackrel{0}{R}_{0 k p i} X^{k} X^{p}+\frac{1}{4} \stackrel{0}{R}_{i k l 0} G_{m} X^{k} X^{l} X^{m}+\frac{1}{6} \stackrel{0}{R}_{0 k l 0} \epsilon_{i m n} \omega^{n} X^{k} X^{l} X^{m} \\
& +\frac{1}{6} \stackrel{0}{R}_{k l i}^{m} \epsilon_{m n p} \omega^{p} X^{k} X^{l} X^{n}+\frac{1}{4} \stackrel{0}{R}_{i k l 0, m} X^{k} X^{l} X^{m}+\cdots, \\
& g_{i j}=\eta_{i j}+\frac{1}{3} \stackrel{0}{R}_{i k p j} X^{k} X^{p}+\frac{1}{12}\left(\stackrel{0}{R}_{i k l 0} \epsilon_{j m n} \omega^{n} X^{k} X^{l} X^{m}+\stackrel{0}{R}_{j k l 0} \epsilon_{i m n} \omega^{n} X^{k} X^{l} X^{m}\right) \\
& +\frac{1}{6} \stackrel{0}{R} i k l j, m X^{k} X^{l} X^{m}+\cdots,
\end{aligned}
$$


where the expression $(\boldsymbol{\omega} \times \boldsymbol{X})_{i}$ denotes $\epsilon_{i j k} \omega^{j} X^{k}$ and $\boldsymbol{G} \boldsymbol{X}:=-G_{i} X^{j}$.

The radius of convergence of series (20) is determined by (see Introduction)

$$
u \ll u_{0}=\min \left\{\frac{1}{|\boldsymbol{G}|}, \quad \frac{1}{|\boldsymbol{\omega}|}, \quad \frac{1}{\left|\stackrel{0}{R}_{\alpha \gamma \delta \beta}\right|^{1 / 2}}, \quad \frac{\left|\stackrel{0}{R}_{\alpha \gamma \delta \beta}\right|}{\left|\stackrel{0}{R}_{\alpha \gamma \delta \beta, i}\right|}\right\} .
$$

Let us compute the integral formula similar to (11). In Fermi coordinates the deviation vectors are given by [2]

$$
\eta_{0}=\delta_{0}^{\mu} \frac{\partial}{\partial X^{\mu}}, \quad \eta_{i}=u \delta_{i}^{\mu} \frac{\partial}{\partial X^{\mu}} .
$$

The straightforward calculation leads to

$$
\begin{gathered}
e_{p}^{(\alpha)}=\delta_{p}^{\alpha}+\frac{1}{u} \int_{0}^{u} d \tau \int_{0}^{\tau} d \tau^{\prime} \tau^{\prime} e_{\lambda}^{(\alpha)} R^{\lambda}{ }_{i j p} \xi^{i} \xi^{j}, \\
e_{0}^{(\alpha)}=\delta_{0}^{\alpha}+\frac{d e_{0}^{0(\alpha)}}{d u} u+\int_{0}^{u} d \tau \int_{0}^{\tau} d \tau^{\prime} e_{\lambda}^{(\alpha)} R^{\lambda}{ }_{i j 0} \xi^{i} \xi^{j} .
\end{gathered}
$$

where

$$
{ }_{e_{\mu}}^{(\alpha)}=\delta_{\mu}^{\alpha}, \quad \frac{d \stackrel{0}{e}_{\mu}^{(\alpha)}}{d u}=\delta_{\mu}^{0} \Omega^{\alpha}{ }_{i} \xi^{i} .
$$

The iterative scheme (10) yields

$$
\begin{aligned}
& e_{\beta}^{(\alpha)}=\sum_{n=0}^{\infty} \stackrel{(n)(\alpha)}{e_{\beta}}, \quad \stackrel{(0)(\alpha)}{e_{\beta}}=\delta_{\beta}^{\alpha}+\delta_{\beta}^{0} \Omega^{\alpha}{ }_{i} \xi^{i} u, \\
& \stackrel{(n+1)(\alpha)}{e_{\beta}}=\frac{1}{u} \int_{0}^{u} d \tau \int_{0}^{\tau} d \tau^{\prime} \tau^{\prime}{\stackrel{(n)(\alpha)}{e_{\lambda}}}^{(\alpha)} R_{\gamma \delta \beta}^{\lambda} \xi^{\gamma} \xi^{\delta},
\end{aligned}
$$

and the radius of convergence of series (23) is determined by conditions

$$
u \ll u_{0}=\min \left\{\frac{1}{|\boldsymbol{G}|}, \quad \frac{1}{|\boldsymbol{\omega}|}, \quad \frac{1}{\mid \stackrel{0}{\left.R_{\alpha \gamma \delta \beta}\right|^{1 / 2}}}\right\} .
$$

In the first approximation one obtains

$$
\begin{gathered}
e_{p}^{(\alpha)}=\delta_{p}^{\alpha}+\frac{1}{u} \int_{0}^{u} d \tau \int_{0}^{\tau} d \tau^{\prime} \tau^{\prime}\left(\delta_{\lambda}^{\alpha}+\delta_{\lambda}^{0} \Omega^{\alpha}{ }_{i} \xi^{i} \tau^{\prime}\right) R^{\lambda}{ }_{j k p} \xi^{j} \xi^{k}+\mathcal{O}\left(R^{2}\right), \\
e_{0}^{(\alpha)}=\delta_{0}^{\alpha}+\Omega^{\alpha}{ }_{i} \xi^{i} u+\int_{0}^{u} d \tau \int_{0}^{\tau} d \tau^{\prime}\left(\delta_{\lambda}^{\alpha}+\delta_{\lambda}^{0} \Omega^{\alpha}{ }_{i} \xi^{i} \tau^{\prime}\right) R^{\lambda}{ }_{j k 0} \xi^{j} \xi^{k}+\mathcal{O}\left(R^{2}\right) .
\end{gathered}
$$

Integrating by parts we combine (25), (26) into one expression

$$
\begin{gathered}
e_{\beta}^{(\alpha)}=\delta_{\beta}^{\alpha}+\delta_{\beta}^{0} \Omega^{\alpha}{ }_{i} \xi^{i} u+\int_{0}^{u} d \tau \int_{0}^{\tau} d \tau^{\prime}\left(\delta_{\lambda}^{\alpha}+\delta_{\lambda}^{0} \Omega^{\alpha}{ }_{i} \xi^{i} \tau^{\prime}\right) R^{\lambda}{ }_{j k \beta} \xi^{j} \xi^{k} \\
-\frac{2}{u} \int_{0}^{u} d \tau \int_{0}^{\tau} d \tau^{\prime} \int_{0}^{\tau^{\prime}} d \tau^{\prime \prime} \delta_{\beta}^{p}\left(\delta_{\lambda}^{\alpha}+\delta_{\lambda}^{0} \Omega^{\alpha}{ }_{i} \xi^{i} \tau^{\prime \prime}\right) R^{\lambda}{ }_{j k p} \xi^{j} \xi^{k}+\mathcal{O}\left(R^{2}\right),
\end{gathered}
$$

and the componets of metric tensor are given by

$$
\begin{array}{r}
g_{\alpha_{\beta}}=\eta_{\alpha_{\beta}}+\delta_{\beta}^{0} \Omega_{\alpha i} \xi^{i} u+\delta_{\alpha}^{0} \Omega_{\beta i} \xi^{i} u+\delta_{\alpha}^{0} \delta_{\beta}^{0} \Omega_{\gamma i} \Omega_{j}^{\gamma} \xi^{i} \xi^{j} u^{2} \\
+\left(\delta_{\alpha}^{\mu}+\delta_{\alpha}^{0} \Omega^{\mu}{ }_{i} \xi^{i} u\right)\left[\int_{0}^{u} d \tau \int_{0}^{\tau} d \tau^{\prime}\left(\eta_{\mu \lambda}+\delta_{\lambda}^{0} \Omega_{\mu i} \xi^{i} \tau^{\prime}\right) R_{j k \beta}^{\lambda} \xi^{j} \xi^{k}\right.
\end{array}
$$




$$
\begin{array}{r}
\left.-\frac{2}{u} \int_{0}^{u} d \tau \int_{0}^{\tau} d \tau^{\prime} \int_{0}^{\tau^{\prime}} d \tau^{\prime \prime} \delta_{\mu}^{p}\left(\eta_{\beta \lambda}+\delta_{\lambda}^{0} \Omega_{\beta i} \xi^{i} \tau^{\prime \prime}\right) R^{\lambda}{ }_{j k p} \xi^{j} \xi^{k}\right] \\
+\left(\delta_{\beta}^{\mu}+\delta_{\beta}^{0} \Omega^{\mu}{ }_{i} \xi^{i} u\right)\left[\int_{0}^{u} d \tau \int_{0}^{\tau} d \tau^{\prime}\left(\eta_{\mu \lambda}+\delta_{\lambda}^{0} \Omega_{\mu i} \xi^{i} \tau^{\prime}\right) R^{\lambda}{ }_{j k \alpha} \xi^{j} \xi^{k}\right. \\
\left.-\frac{2}{u} \int_{0}^{u} d \tau \int_{0}^{\tau} d \tau^{\prime} \int_{0}^{\tau^{\prime}} d \tau^{\prime \prime} \delta_{\mu}^{p}\left(\eta_{\alpha \lambda}+\delta_{\lambda}^{0} \Omega_{\alpha i} \xi^{i} \tau^{\prime \prime}\right) R^{\lambda}{ }_{j k p} \xi^{j} \xi^{k}\right]+\mathcal{O}\left(R^{2}\right),
\end{array}
$$

This integral formula agrees with the series (20).

The transformation from arbitrary coordinate system $\left\{x^{\mu}\right\}$ to Fermi one takes the form

$$
x^{\mu}=x^{\mu}\left(X^{0}\right)+\Lambda_{i}^{\mu}\left(X^{0}\right) X^{i}+\cdots,
$$

and in the coordinates $\left\{x^{\mu}\right\}$ one can write Eq.(27) as

$$
\begin{aligned}
e_{\beta}^{(\alpha)}= & \delta_{\beta}^{\alpha}+\delta_{\beta}^{0} \Omega^{\gamma}{ }_{i} \Lambda_{\gamma}^{-1 \alpha} \xi^{i} u+\int_{0}^{u} d \tau \int_{0}^{\tau} d \tau^{\prime}\left(\Lambda_{\gamma}^{-1 \alpha}+\Lambda_{\lambda}{ }^{0} \Lambda_{\gamma}^{-1 \alpha} \Omega^{\gamma}{ }_{i} \xi^{i} \tau^{\prime}\right) R^{\lambda}{ }_{\delta \kappa \beta} \Lambda_{j}{ }^{\delta} \Lambda_{k}{ }^{\kappa} \xi^{j} \xi^{k} \\
& -\frac{2}{u} \int_{0}^{u} d \tau \int_{0}^{\tau} d \tau^{\prime} \int_{0}^{\tau^{\prime}} d \tau^{\prime \prime} \delta_{\beta}^{p}\left(\Lambda_{\gamma}^{-1 \alpha}+\Lambda_{\lambda}{ }^{0} \Lambda_{\gamma}^{-1 \alpha} \Omega^{\gamma}{ }_{i} \xi^{i} \tau^{\prime \prime}\right) R^{\lambda}{ }_{\delta \kappa p} \Lambda_{j}{ }^{\delta} \Lambda_{k}{ }^{\kappa} \xi^{j} \xi^{k}+\mathcal{O}(R),
\end{aligned}
$$

where it is used the same notation $R^{\alpha}{ }_{\beta \gamma \delta}$ for the curvature tensor in the coordinate system $x^{\mu}$ and

$$
R_{\mu \nu \lambda}^{\alpha}=R_{\mu \nu \lambda}^{\alpha}\left(x^{\beta}\left(X^{0}\right)+\Lambda_{i}^{\beta}\left(X^{0}\right) \xi^{i} \tau^{\prime \prime}\right)
$$

while the integration is performed.

\section{WEAK PLANE-WAVE METRIC IN FERMI NORMAL COORDINATES}

We compute the plane-wave metric in Fermi normal coordinates for a geodesic observer in the weak gravitationalwave field, whose metric tensor is usually written in synchronous coordinates,

$$
d s^{2}=\eta_{\mu \nu} d x^{\mu} d x^{\nu}+h_{a b} d x^{a} d x^{b},
$$

where $a$ and $b$ run from 1 to 2 while

$$
h_{a b}=h_{a b}(t-z), h_{22}=-h_{11}=h_{+}, h_{12}=h_{\times} .
$$

Using the definition of the Riemann tensor

$$
R_{\mu \nu \lambda \sigma}=\frac{1}{2}\left(h_{\nu \lambda, \mu, \sigma}+h_{\mu \sigma, \nu l a m b d a}-h_{\mu \lambda, \nu, \sigma}-h_{\nu \sigma, \mu, \lambda}\right),
$$

one finds that in the linearized theory non-zero components of Riemann tensor are

$$
\begin{aligned}
R_{3 a b 3} & =R_{0 a b 0}=-R_{3 a b 0}=\frac{1}{2} \ddot{h}_{a b}, \\
R_{\mu 22 \nu} & =-R_{\mu 11 \nu}, R_{\mu 12 \nu}=R_{\mu 21 \nu},
\end{aligned}
$$

dot being derivative with respect to $t$.

The next step is to choose an orthonormal frame along the world line $\gamma$ of observer. Taking into account the transformation to the Fermi normal coordinates $x^{\mu}=X^{\mu}+\mathcal{O}\left(h_{a b}\right)$ and that the timelike base vector must be the tangent $\partial / \partial t$, one obtains

$$
\begin{array}{ll}
\left.\mathbf{e}_{(0)}\right|_{\gamma}=\partial / \partial T=\partial / \partial t, & \left.\mathbf{e}_{(1)}\right|_{\gamma}=\partial / \partial X=\partial / \partial x, \\
\left.\mathbf{e}_{(2)}\right|_{\gamma}=\partial / \partial Y=\partial / \partial y, & \left.\mathbf{e}_{(3)}\right|_{\gamma}=\partial / \partial Z=\partial / \partial z,
\end{array}
$$

where $X^{0}=T, X^{1}=X, X^{2}=Y, X^{3}=Z$ are setted.

Using (28) we compute the metric tensor in the Fermi normal coordinates. The result is 


$$
\begin{array}{r}
g_{00}=1+H_{a b} X^{a} X^{b}, \\
g_{a b}=\eta_{a b}+F_{a b} Z^{2}, \\
g_{0 a}=\frac{1}{2}\left(H_{a b}+F_{a b}\right) X^{b} Z, \\
g_{03}=-\frac{1}{2}\left(H_{a b}+F_{a b}\right) X^{a} X^{b}, \\
g_{33}=-1+F_{a b} X^{a} X^{b}, \\
g_{a 3}=-F_{a b} X^{b} Z,
\end{array}
$$

where

$$
\begin{array}{r}
H_{a b}=\frac{1}{Z^{2}}\left(h_{a b}(T-Z)-h_{a b}(T)+Z \dot{h}_{a b}(T)\right), \\
F_{a b}=H_{a b}-\frac{2}{Z^{3}} \int_{0}^{Z} H_{a b}(T, Y) Y^{2} d Y .
\end{array}
$$

It is easy to see that ${H^{\prime}}^{\prime}{ }_{a b}=-\dot{F}_{a b}, H_{a b}(T, 0)=3 F_{a b}(T, 0)=(1 / 2) \ddot{h}_{a b}(T)$ and

$$
F_{a b}=\frac{1}{2}\left(\left(H_{a b}-F_{a b}\right) Z\right)^{\prime},
$$

here and below dot being derivative with respect to $T$ and prime derivative with respect to $Z$. The nonvanishing components of Christoffel symbols are found to be

$$
\begin{gathered}
\Gamma_{0 b}^{a}=\frac{1}{2} H_{a b}^{\prime} Z^{2}, \quad \Gamma_{00}^{a}=H_{a b} X^{b}-\frac{1}{2}\left(\dot{H}_{a b}-H_{a b}^{\prime}\right) X^{b} Z, \\
\Gamma_{33}^{a}=2 F_{a b} X^{b}+F_{a b}^{\prime} X^{b} Z, \quad \Gamma_{3 b}^{a}=-F_{a b} Z-\frac{1}{2} F_{a b}^{\prime} Z^{2} \\
\Gamma_{03}^{a}=-H_{a b} X^{b}-H_{a b}^{\prime} Z X^{b}, \quad \Gamma_{0 a}^{0}=\Gamma_{0 a}^{3}=H_{a b} X^{b}, \\
\Gamma_{a b}^{0}=\Gamma_{a b}^{3}=2 F_{a b} Z+\frac{1}{2} F_{a b}^{\prime} Z^{2}, \quad \Gamma_{a 3}^{3}=-F_{a b} X^{b}, \\
\Gamma_{00}^{0}=\Gamma_{00}^{3}=\frac{1}{2} \dot{H}_{a b} X^{a} X^{b}, \quad \Gamma_{33}^{0}=-\frac{1}{2} F_{a b}^{\prime} X^{a} X^{b}, \\
\Gamma_{03}^{0}=\Gamma_{03}^{3}=\frac{1}{2} H_{a b}^{\prime} X^{a} X^{b}, \quad \Gamma_{33}^{3}=-\frac{1}{2} F_{a b}^{\prime} X^{a} X^{b},
\end{gathered}
$$

and the computation of the non-zero components of Riemann tensors in Fermi normal coordinates yields

$$
\begin{array}{r}
R_{3 a b 3}=R_{0 a b 0}=-R_{3 a b 0} \\
=\frac{1}{2}\left\{\ddot{F}_{a b} Z^{2}+2 H_{a b}-\left(\dot{H}_{a b}+\dot{F}_{a b}\right) Z\right\} .
\end{array}
$$

This leads to

$$
R_{3 a b 3}=R_{0 a b 0}=-R_{3 a b 0}=\frac{1}{2} \ddot{h}_{a b}(T-Z) .
$$

Thus in Fermi coordinates the curvature is the same function $(1 / 2) \ddot{h}_{a b}$, but depending on $(T-Z)$.

Plane monochromaric gravitational wave. The only nonzero components $h_{a b}=A_{a b} \sin [k(t-z)]$ of wave amplitudes are

$$
\begin{gathered}
h_{11}=-h_{22}=A_{+} \sin [k(t-z)], \\
h_{12}=h_{21}=A_{\times} \sin [k(t-z)] .
\end{gathered}
$$

Computation yields

$$
\begin{array}{r}
H_{a b}=k^{2} A_{a b}\left[\cos (k T) \frac{k Z-\sin (k Z)}{(k Z)^{2}}+\sin (k T) \frac{\cos (k Z)-1}{(k Z)^{2}}\right] \\
F_{a b}=\left\{\cos (k T)\left[\frac{k Z-\sin (k Z)}{(k Z)^{2}}-2 \frac{\cos (k Z)-1+(k Z)^{2} / 2}{(k Z)^{3}}\right]\right. \\
\left.+\sin (k T)\left[\frac{\cos (k Z)-1}{(k Z)^{2}}+2 \frac{k Z-\sin (k Z)}{(k Z)^{3}}\right]\right\}
\end{array}
$$


This leads to

$$
\begin{aligned}
& g_{00}=1+k^{2} A_{a b} X^{a} X^{b}\left[\cos (k T) \frac{k Z-\sin (k Z)}{(k Z)^{2}}+\sin (k T) \frac{\cos (k Z)-1}{(k Z)^{2}}\right] \text {, } \\
& g_{03}=k^{2} A_{a b} X^{a} X^{b}\left\{\cos (k T)\left[\frac{\sin (k Z)-k Z}{(k Z)^{2}}+\frac{\cos (k Z)-1+(k Z)^{2} / 2}{(k Z)^{3}}\right]\right. \\
& \left.+\sin (k T)\left[\frac{\sin (k Z)-k Z}{(k Z)^{2}}-\frac{\cos (k Z)-1}{(k Z)^{2}}\right]\right\}, \\
& g_{0 a}=-k^{2} A_{a b} X^{b} Z\left\{\cos (k T)\left[\frac{\sin (k Z)-k Z}{(k Z)^{2}}+\frac{\cos (k Z)-1+(k Z)^{2} / 2}{(k Z)^{3}}\right]\right. \\
& \left.+\sin (k T)\left[\frac{\sin (k Z)-k Z}{(k Z)^{2}}-\frac{\cos (k Z)-1}{(k Z)^{2}}\right]\right\}, \\
& g_{a b}=\eta_{a b}+k^{2} A_{a b} Z^{2}\left\{\cos (k T)\left[\frac{k Z-\sin (k Z)}{(k Z)^{2}}-2 \frac{\cos (k Z)-1+(k Z)^{2} / 2}{(k Z)^{3}}\right]\right. \\
& \left.+\sin (k T)\left[\frac{\cos (k Z)-1}{(k Z)^{2}}+2 \frac{k Z-\sin (k Z)}{(k Z)^{3}}\right]\right\} \\
& g_{a 3}=-k^{2} A_{a b} X^{b} Z\left\{\cos (k T)\left[\frac{k Z-\sin (k Z)}{(k Z)^{2}}-2 \frac{\cos (k Z)-1+(k Z)^{2} / 2}{(k Z)^{3}}\right]\right. \\
& \left.+\sin (k T)\left[\frac{\cos (k Z)-1}{(k Z)^{2}}+2 \frac{k Z-\sin (k Z)}{(k Z)^{3}}\right]\right\} \\
& g_{33}=-1+k^{2} A_{a b} X^{a} X^{b}\left\{\cos (k T)\left[\frac{k Z-\sin (k Z)}{(k Z)^{2}}-2 \frac{\cos (k Z)-1+(k Z)^{2} / 2}{(k Z)^{3}}\right]\right. \\
& \left.+\sin (k T)\left[\frac{\cos (k Z)-1}{(k Z)^{2}}+2 \frac{k Z-\sin (k Z)}{(k Z)^{3}}\right]\right\},
\end{aligned}
$$

where we set

$$
\begin{array}{r}
A_{a b} X^{b}=A_{+}\left(\delta_{a}^{1} X-\delta_{a}^{2} Y\right)+A_{\times}\left(\delta_{a}^{1} Y+\delta_{a}^{2} X\right), \\
A_{a b} X^{a} X^{b}=A_{+}\left(X^{2}-Y^{2}\right)+2 A_{\times} X Y .
\end{array}
$$

Our results agree with the metric obtained in 13 .

Geodesic deviation. Let us consider the geodesic deviation of two neighbouring geodesics in the gravitational field of a plane weak wave. The separation vector $\eta$ satisfies a geodesic deviation equation (6), which reads

$$
\frac{d^{2} \eta^{(\alpha)}}{d \lambda^{2}}=R_{(\beta)(\gamma)(\delta)}^{(\alpha)} \zeta^{(\beta)} \zeta^{(\gamma)} \eta^{(\delta)}
$$

$\lambda$ being a canonical parameter along the geodesic and $\zeta$ a tangent vector to it. The solution of this equation is given by

$$
\eta^{(\alpha)}=\eta_{0}^{\alpha}+\int_{0}^{\lambda} d \tau \int_{0}^{\tau} d \tau^{\prime} R_{\beta \gamma \delta}^{\alpha} \stackrel{\zeta}{\beta}^{\beta} \check{\zeta}^{\gamma} \eta_{0}^{\delta},+\mathcal{O}\left(R^{2}\right)
$$

where we set $\eta_{0}^{\alpha}=\eta^{\alpha}(0)$ and integration being performed along the basic geodesic line canonically parametrized by $\lambda$

The separation magnitude is found to be

$$
l=l_{0}-\int_{0}^{\lambda} d \tau \int_{0}^{\tau} d \tau^{\prime} R_{\alpha \beta \gamma \delta} \zeta^{\beta} \zeta^{0}{ }^{\gamma} \eta_{0}^{\delta} \eta_{0}^{\alpha},
$$

Now let us consider the geodesic deviation of two neighbouring particles $A$ and $B$ assuming that the the origin of Fermi reference frame attached to $A^{\prime}$ s geodesic and for $T=0$ the particles are in the plane $Z=0$. In this case the components of the separation vector are nothing but the coordinates of particle $\mathrm{B}: \eta^{\alpha}=X^{\alpha}, \zeta^{\beta}=\delta_{0}^{\beta}$ and the canonical parameter $\lambda$ being a proper time $\tau$ along the geodesic line of the observer. Then (53) reads 


$$
l=l_{0}-\frac{1}{l_{0}} \int_{0}^{\lambda} d \tau \int_{0}^{\tau} d \tau^{\prime} R_{0 a b 0} X^{a} X^{b} .
$$

This yields

$$
l=l_{0}-\frac{1}{l_{0}} h_{a b}(T) X^{a} X^{b}
$$

and for the plane monochromatic gravitational wave one obtains the well known result $[4$

$$
l=l_{0}-\frac{l_{0}}{2}\left(A_{+} \cos 2 \varphi+A_{\times} \sin 2 \varphi\right) \sin (k T) .
$$

\section{ACKNOWLEDGEMENTS}

It is a pleasure to acknowledge numerous conversations with N. V. Mitskievich. This work was supported by CONACyT grant 1626 P-E.

\section{APPENDIX A}

Here we shall obtain the formula (Eq.(13) in the text)

$$
\nabla_{\partial_{\lambda}} e_{(\nu)}^{\mu}=-\frac{1}{u} \int_{0}^{u} \tau d \tau R_{\nu \lambda \delta}^{\mu} \stackrel{0}{\xi^{\delta}}+\mathcal{O}(R) .
$$

Let us start with the Taylor expansion for tetrad in the neighbourhood $V\left(p_{0}\right)$

$$
e_{(\nu)}^{\mu}=\delta_{\nu}^{\mu}+\frac{d \stackrel{0}{e}_{(\nu)}^{\mu}}{d u} u+\frac{1}{2 !} \frac{d^{2} \stackrel{0}{e}_{(\nu)}^{\mu}}{d u^{2}} u^{2}+\frac{1}{3 !} \frac{d^{3} \stackrel{0}{e}_{(\nu)}^{\mu}}{d u^{3}} u^{3}+\cdots,
$$

$u$ being the canonical parameter along geodesics starting in the point $p_{0}$. Using in the Riemann normal coordinates the equation of parallel propagation

$$
\frac{d e_{(\nu)}^{\mu}}{d u}+\Gamma_{\sigma \lambda}^{\mu} e_{(\nu)}^{\sigma} \xi^{\lambda}=0
$$

we rewrite (57) as

$$
e_{(\nu)}^{\mu}=\delta_{\nu}^{\mu}-\frac{1}{2 !} \stackrel{0}{\Gamma}_{\nu \sigma, \gamma}^{\mu} X^{\sigma} X^{\gamma}-\frac{1}{3 !} \stackrel{0}{\Gamma}_{\nu \sigma, \gamma, \delta}^{\mu} X^{\sigma} X^{\gamma} X^{\delta}+\cdots+\mathcal{O}\left(R^{2}\right) .
$$

The expansion of the connection coefficients is given by

$$
\Gamma_{\nu \lambda}^{\mu}=\stackrel{0}{\Gamma^{\mu}}{ }_{\nu \lambda, i} X^{i}+\frac{1}{2 !} \stackrel{0}{\Gamma}_{\nu \lambda, i, l}^{\mu} X^{i} X^{l}+\cdots .
$$

Applying Eq. (59), (60) we obtain the following series for the covariant derivatives of tetrad

$$
\begin{aligned}
\nabla_{\partial_{\lambda}} e_{(\nu)}{ }^{\mu}=\stackrel{0}{\Gamma}_{\nu \lambda, \sigma}^{\mu} X^{\sigma} & -\stackrel{0}{\Gamma}_{\nu(\lambda, \sigma)}^{\mu} X^{\sigma}+\frac{1}{2 !}\left(\stackrel{0}{\Gamma}_{\nu \lambda, \sigma, \gamma}^{\mu}-\stackrel{0}{\Gamma}_{\nu(\lambda, \sigma, \gamma)}\right) X^{\sigma} X^{\gamma} \\
& +\frac{1}{3 !}\left(\stackrel{0}{\Gamma}_{\nu \lambda, \sigma, \gamma, \delta}^{\mu}-\stackrel{0}{\Gamma}_{\nu(\lambda, \sigma, \gamma, \delta)}^{\mu}\right) X^{\sigma} X^{\gamma} X^{\delta}+\mathcal{O}\left(R^{2}\right) .
\end{aligned}
$$

Now using the relations

$$
\begin{array}{r}
\frac{1}{2} \stackrel{0}{R}^{\mu}{ }_{\nu \sigma \lambda} X^{\sigma}=\left(\stackrel{0}{\Gamma}_{\nu \lambda, \sigma}-\stackrel{0}{\Gamma}_{\nu(\lambda, \sigma)}^{\mu}\right) X^{\sigma}, \\
\frac{2}{3}{\stackrel{0}{R^{\mu}}}_{\nu \sigma \lambda, \gamma} X^{\sigma} X^{\gamma}=\left(\stackrel{0}{\Gamma}_{\nu \lambda, \sigma, \gamma}^{\mu}-\stackrel{0}{\Gamma}_{\nu(\lambda, \sigma, \gamma)}^{\mu}\right) X^{\sigma} X^{\gamma}, \\
\frac{3}{4} \stackrel{0}{R}_{\nu \sigma \lambda, \gamma \delta} X^{\sigma} X^{\gamma} X^{\delta}=\left(\stackrel{0}{\Gamma}_{\nu \lambda, \sigma, \gamma, \delta}-\stackrel{0}{\Gamma}_{\nu(\lambda, \sigma, \gamma, \delta)}^{\mu}\right) X^{\sigma} X^{\gamma} X^{\delta}, \quad \text { etc }
\end{array}
$$


one can write the expansion (61) as

$$
\begin{array}{r}
\nabla_{\partial_{\lambda}} e_{(\nu)}^{\mu}=\frac{1}{2}{\stackrel{0}{R^{\mu}}}_{\nu \sigma \lambda} X^{\sigma}+\frac{2}{3 !} \stackrel{0}{R^{\mu}}{ }_{\nu \sigma \lambda, \gamma} X^{\sigma} X^{\gamma}+\frac{3}{4 !} \stackrel{0}{R}_{\nu \sigma \lambda, \gamma}^{\mu} X^{\sigma} X^{\gamma} X^{\delta}+\cdots \\
+\frac{n}{(n+1) !} \stackrel{0}{R^{\mu}}{ }_{\nu \gamma_{1} \lambda, \gamma_{2}, \cdots, \gamma_{n}} X^{\gamma_{1}} X^{\gamma_{2}} \cdots X^{\gamma_{n}}+\cdots+\mathcal{O}\left(R^{2}\right) .
\end{array}
$$

It is convenient to present this series in the form

$$
\nabla_{\partial_{\lambda}} e_{(\nu)}^{\mu}=\sum_{n=0}^{\infty} \frac{d^{n}\left(\stackrel{0}{R}^{\mu}{ }_{\nu \sigma \lambda} \xi^{\sigma}\right)}{d u^{n}} \frac{u^{n+1}}{(n+2)(n !)}+\mathcal{O}\left(R^{2}\right)
$$

Straightforward calculation yields the following integral representation of Eq.(61):

$$
\nabla_{\partial_{\lambda}} e_{(\nu)}^{\mu}=-\frac{1}{u} \int_{0}^{u} R_{\nu \lambda \sigma}^{\mu} \xi^{\sigma} \tau d \tau+\mathcal{O}\left(R^{2}\right)
$$

Integrating by parts it found to be

$$
\nabla_{\partial_{\lambda}} e_{(\nu)}^{\mu}=-\int_{0}^{u} R_{\nu \lambda \sigma}^{\mu} \xi^{\sigma} d \tau+\frac{1}{u} \int_{0}^{u} d \tau \int_{0}^{\tau} d \tau^{\prime} R^{\mu}{ }_{\nu \lambda \sigma} \xi^{\sigma}+\mathcal{O}\left(R^{2}\right)
$$

\section{APPENDIX B}

Here we shall obtain the series (Eq.(19) in the text)

$$
\begin{array}{r}
e_{0}^{(\mu)}=\delta_{0}^{\mu}+\Omega^{\mu}{ }_{i} X^{i}+\frac{1}{2} \stackrel{0}{R}^{\mu}{ }_{i j 0} X^{i} X^{j}+\frac{1}{6} \stackrel{0}{R}_{0 i j 0} \Omega^{\mu}{ }_{k} X^{i} X^{j} X^{k}+\frac{1}{6} \stackrel{0}{R}^{\mu}{ }_{i j 0, k} X^{i} X^{j} X^{k}+\cdots \\
e_{p}^{(\mu)}=\delta_{p}^{\mu}+\frac{1}{6} \stackrel{0}{R}^{\mu}{ }_{i j p} X^{i} X^{j}+\frac{1}{12} \stackrel{0}{R}^{0}{ }_{i j p} \Omega^{\mu}{ }_{k} X^{i} X^{j} X^{k}+\frac{1}{12} \stackrel{0}{R}^{\mu}{ }_{i j p, k} X^{i} X^{j} X^{k}+\cdots
\end{array}
$$

We start with the Taylor expansion for tetrad in a world tube surrounding the world line $\gamma$ of Fermi observer:

$$
e_{\mu}^{(\nu)}=\delta_{\nu}^{\mu}+\frac{d e_{\mu}^{(\nu)}(0)}{d u} u+\frac{1}{2 !} \frac{d^{2} e_{\mu}^{(\nu)}(0)}{d u^{2}} u^{2}+\frac{1}{3 !} \frac{d^{3} e_{\mu}^{(\nu)}(0)}{d u^{3}} u^{3}+\cdots
$$

$u$ being the canonical parameter along spacelike geodesics ortogonal to $\gamma$. Using in Fermi coordinates the equation of parallel propagation

$$
\frac{d e_{\mu}^{(\nu)}}{d u}-\Gamma_{\mu i}^{\sigma} e_{\sigma}^{(\nu)} \xi^{i}=0
$$

we rewrite Eq. 66) as

$$
\begin{aligned}
& e_{\mu}^{(\nu)}=\delta_{\mu}^{\nu}+\stackrel{0}{\Gamma}_{\mu i}^{\nu} X^{i}+\frac{1}{2 !}\left(\stackrel{0}{\Gamma}^{\nu}{ }_{\mu i, l}+\stackrel{0}{\Gamma}^{\lambda}{ }_{\mu i} \stackrel{0}{\Gamma}^{\nu}{ }_{\lambda l}\right) X^{i} X^{l}
\end{aligned}
$$

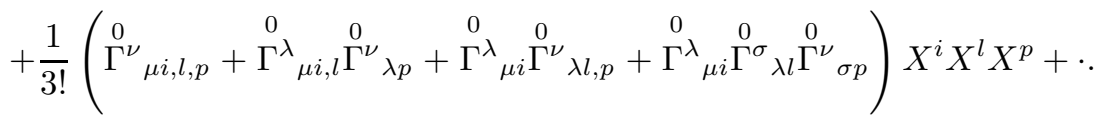

For computing of the connection coefficients and its derivatives one needs the spacelike geodesic deviation equation

$$
\frac{D^{2} \eta^{\mu}}{d u^{2}}-R^{\mu}{ }_{i j \nu} \xi^{i} \xi^{j} \eta^{\nu}=0
$$

It is convenient to write it as 


$$
\frac{d^{2} \eta^{\mu}}{d u^{2}}+\frac{d \Gamma_{\nu i}^{\mu}}{d u} \xi^{i} \eta^{\nu}+2 \Gamma_{\nu i}^{\mu} \xi^{i} \frac{d \eta^{\nu}}{d u}+\Gamma_{\nu i}^{\lambda} \Gamma_{\lambda j}^{\mu} \xi^{i} \xi^{j} \eta^{\nu}=R^{\mu}{ }_{i j \nu} \xi^{i} \xi^{j} \eta^{\nu}
$$

Using deviation vectors $\eta_{(i)}^{\mu}=u \delta_{i}^{\mu}$ and $\eta_{0}^{\mu}=\delta_{0}^{\mu}$, we write (69) as follows:

$$
\begin{array}{r}
\frac{d \Gamma_{p i}^{\mu}}{d u} \xi^{i} u+2 \Gamma_{p i}^{\mu} \xi^{i}+\Gamma_{p i}^{\lambda} \Gamma_{\lambda j}^{\mu} \xi^{i} \xi^{j} u=R^{\mu}{ }_{i j p} \xi^{i} \xi^{j} u \\
\frac{d \Gamma_{0 i}^{\mu}}{d u} \xi^{i}+\Gamma_{0 i}^{\lambda} \Gamma_{\lambda j}^{\mu} \xi^{i} \xi^{j}=R^{\mu}{ }_{i j 0} \xi^{i} \xi^{j} .
\end{array}
$$

From Eq.(18) one obtains $\stackrel{0}{\Gamma}_{\mu \nu}^{\lambda}=\delta_{\mu}^{0} \Omega^{\lambda}{ }_{\nu}$. These conditions together with (70), (71) yield

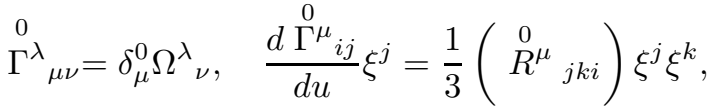

$$
\begin{aligned}
& \frac{d \stackrel{0}{\Gamma^{\mu}}{ }_{0 i}}{d u} \xi^{i}=\left(\stackrel{0}{R^{\mu}}{ }_{i k 0}-\Omega^{0}{ }_{i} \Omega^{\mu}{ }_{k}\right) \xi^{i} \xi^{k}, \\
& \frac{d^{2} \stackrel{0}{\Gamma}^{\mu}{ }_{o i}}{d u^{2}} \xi^{i}=\left({\stackrel{0}{R^{\mu}}}_{i j 0, k}-\stackrel{0}{R}_{0 i j 0} \Omega^{\mu}{ }_{k}-\stackrel{0}{R}^{\mu}{ }_{i j 0} \Omega_{0 k}\right. \\
& \left.-\frac{1}{3} \stackrel{0}{R}^{\mu}{ }_{i j p} \Omega^{p}{ }_{k}+2 \Omega^{\mu}{ }_{i} \Omega_{0 j} \Omega_{0 k}\right) \xi^{i} \xi^{j} \xi^{k}, \\
& \frac{d^{2} \stackrel{0}{\Gamma}^{\mu}{ }_{i j}}{d u^{2}} \xi^{j}=\left(\frac{1}{2} \stackrel{0}{R}^{\mu}{ }_{j k i, p}-\frac{1}{6} \stackrel{0}{R}_{0 j k i} \Omega^{\mu}{ }_{p}\right) \xi^{j} \xi^{k} \xi^{p} .
\end{aligned}
$$

Finally, applying $(72-75)$ and (68) we compute

$$
\begin{array}{r}
e_{0}^{(\mu)}=\delta_{0}^{\mu}+\Omega^{\mu}{ }_{i} X^{i}+\frac{1}{2} \stackrel{0}{R}^{\mu}{ }_{i j 0} X^{i} X^{j} \\
+\frac{1}{6} \stackrel{0}{R}_{0 i j 0} \Omega^{\mu}{ }_{k} X^{i} X^{j} X^{k}+\frac{1}{6} \stackrel{0}{R}^{\mu}{ }_{i j 0, k} X^{i} X^{j} X^{k}+\cdots, \\
e_{p}^{(\mu)}=\delta_{p}^{\mu}+\frac{1}{6} \stackrel{0}{R}^{\mu}{ }_{i j p} X^{i} X^{j}+\frac{1}{12} \stackrel{0}{R}^{0}{ }_{i j p} \Omega^{\mu}{ }_{k} X^{i} X^{j} X^{k} \\
+\frac{1}{12} \stackrel{0}{R}^{\mu}{ }_{i j p, k} X^{i} X^{j} X^{k}+\cdots
\end{array}
$$


[1] Fermi E 1922 Atti Acad. Naz. Lincei Rend. Cl. Sci. Fiz. Mat. Nat. 31 21, 51

[2] Manasse F K and Misner C W 1963 J. Math. Phys. 4, 735

[3] Synge J L 1960 Relativity: The General Theory (North-Holland, Amsterdam)

[4] Misner C W, Thorne K S and Wheeler J A 1973 Gravitation (Freeman, New York)

[5] Mitskievich N V and Nesterov A I 1976 Izv. Vuzov. (Fisica). No. 992

[6] Ni W -T and Zimmermann M 1978 Phys. Rev. D 171473

[7] Li W -Q and Ni W -T 1979 J. Math. Phys. 201473

[8] Li W -Q and Ni W -T 1979 J. Math. Phys. 201925

[9] Marzlin K -P 1994 Phys. Rev. D 50888

[10] Mitskievich N V and Nesterov A I 1995 Gen. Relat. Grav. 27361

[11] Mitskievich N V and Nesterov A I 1996 Int. J. Theor. Phys. 352677

[12] Arfken J 1968 Mathematical Methods for Physicists (Academic Press, New York)

[13] Faraoni V 1992 Nuovo Cim. B 43631 